\title{
A Resolution of St. Petersburg Paradox
}

\author{
V.I. Yukalov \\ Bogolubov Laboratory of Theoretical Physics, JINR, Dubna 141980, Russia \\ E-mail: yukalov@theor.jinr.ru
}

\begin{abstract}
The St. Petersburg paradox is the oldest paradox in decision theory and has played a pivotal role in the introduction of increasing concave utility functions embodying risk aversion and decreasing marginal utility of gains. All attempts to resolve it have considered some variants of the original set-up, but the original paradox has remained unresolved, while the proposed variants have introduced new complications and problems. Here a rigorous mathematical resolution of the St. Petersburg paradox is suggested based on a probabilistic approach to decision theory.
\end{abstract}

\section{JEL classification: G11, G12}

Keywords: Bernoulli game, St. Petersburg paradox, probabilistic decision theory

Declarations of interest: none 


\section{Introduction}

The famous St. Petersburg paradox is, probably, the oldest paradox in decision theory, and can be said to have promoted the birth of modern decision theory itself. It was invented by Nicolas Bernoulli in 1713 and discussed in his private letters. The paradox was formally stated by his cousin Daniel Bernoulli (1738), who worked in St. Petersburg. The paradox and the suggested solution were published in the Proceedings of the Imperial Academy of Sciences of St. Petersburg (Bernoulli (1738)).

In economics, the St. Petersburg paradox has played a particularly important role in pointing out situations in which supposedly rational decisions based on expected gains or even expected increasing utilities are not endorsed by real rational human decision makers. The St. Petersburg paradox has opened a flood of attempts to solve it, which turn out all to modify it in one way or another. The most important change involves the introduction of a concave utility function which, in the words of Cramer (1728), captures the concept that "men of good sense estimate money in proportion to the usage that they may make of it" (and not necessarily in proportion to its quantity). Motivated by the St. Petersburg paradox, the introduction of concave utility functions, which embody risk aversion and decreasing marginal utility of gains, remains the central pillar of modern economic theory.

However, it turns out that the solution in terms of utility functions of Cramer (1728) and Bernoulli (1738) is not completely satisfactory since, as is stressed below, a slight change in the formulation makes the paradox reappear. This problem and the many proposed solutions have been revisited recently by Clark (2002) and by Martin (2008). Thus, Clark states "This seems to be one of those paradoxes we have to swallow". Or, as Martin concludes "The St. Petersburg result is strange... The appropriate reaction might just be to try to accept the strange result". As the suggested solutions all modify the initial problem, it is important to emphasize that the original paradox has remained unresolved.

Here, we propose a resolution of the St. Petersburg paradox based on the introduction of stochasticity of the decision making problem to address the paradox. Stochasticity is argued to be the natural intrinsic feature of human behavior.

To be precise, first of all, in Section 2, we identify the origin of the St. Petersburg paradox. In Section 3, we briefly summarize the most known attempts to avoid it by changing the original formulation and explain why such attempts do not resolve the paradox in its general form. In Section 4, we briefly mention the models used in stochastic decision making and discuss the justification of stochastic approach. Then, in Section 5, we formulate the probabilistic approach to decision theory to be used and in Section 6 show how the paradox becomes resolved in the frame of this approach. In the probabilistic approach there exists a belief (disbelief) parameter whose estimation for the case of the St. Petersburg paradox is considered in Section 7. The role of the trial prior probability is discussed in Section 8. The existence of repeated Bernoulli games is mentioned in Section 9, and in Section 10, the so-called inverse St. Petersburg paradox is investigated. Section 11 concludes. 


\section{Origin of St. Petersburg Paradox}

First, we have to correctly formulate the problem in order to understand where the paradox is located. We emphasize that the game, which the paradox stems from, is a correctly posed mathematical problem. To resolve a problem does not mean to change it, but this means to find a week point in its attempted solution and to change the solution. The essence of the St. Petersburg paradox is not in the formulation of the game, but in the definition of what has to be accepted as an optimal lottery.

In the St. Petersburg paradox, one meets the possibility of infinite number of actions, such as coin tosses. Dealing with infinity in applied sciences one keeps in mind the notion of potential infinity that is assumed to be defined as a limit of a sequence. Potential infinity is treated not as an actual number, but as a trend dealing with asymptotically large numbers, which are such large numbers that, though being finite, are greater than any number given apriori (Poincaré (1902), Moore (1990), Rucker (1995)).

Below, following Samuelson $(1960,1977)$, we accept this understanding of an infinite Bernoulli game as a limit of a sequence of finite games and consider the sequence of lotteries. Only then it is possible to mathematically correctly formalize how a person makes a decision to play a toss game and how much the game would be worth.

\subsection{Original Bernoulli game}

It is necessary to distinguish the Bernoulli game lying at the heart of the St. Petersburg paradox and the paradox as such.

The game, as it has been originally formulated by Bernoulli (1738), is defined as follows. Paying a fixed fee to enter the game, a player tosses a fair coin repeatedly until tails first appear, ending the game. If tails appear for the first time at the $n$-th toss, the player wins $x_{n}=2^{n}$ monetary units. Thus, the player wins 2 monetary units if tails appear on the first toss, $2^{2}$ units, if on the second, $2^{3}$ units, if on the third, and so on. The gain of $2^{n}$ units occurs therefore with the probability $1 / 2^{n}$. The cost of the ticket to enter the game is proportional to the number of allowed tosses. The main question is: How much should a player pay for the ticket to enter the game? Or in other words: How many tosses should a player buy?

Remark. The nature of the monetary units is not important. It could be Dollars, Euros, Francs, or any other units. Therefore, for the sake of brevity, we shall omit in what follows the use of the term "monetary units", when this cannot lead to confusion.

In mathematical parlance, the above game reads as follows (Samuelson $(1960,1977)$ ). There is a set of lotteries $\left\{L_{n}: n=1,2, \ldots, \infty\right\}$ labeled according to the number of allowed tosses, so that the first lottery is

$$
L_{1}=\left\{2, \frac{1}{2} \mid 0, \frac{1}{2}\right\},
$$

the second lottery is

$$
L_{2}=\left\{2, \frac{1}{2}\left|2^{2}, \frac{1}{2^{2}}\right| 0, \frac{1}{2^{2}}\right\} \text {, }
$$


and so on, with the $n$-th lottery being

$$
L_{n}=\left\{2, \frac{1}{2}\left|2^{2}, \frac{1}{2^{2}}\right| \ldots\left|2^{n}, \frac{1}{2^{n}}\right| 0, \frac{1}{2^{n}}\right\},
$$

while, as has been stressed above, the lottery $L_{\infty}$ implies the limit of the series $\left\{L_{n}\right\}$ for $n \rightarrow \infty$.

The probability distribution of each lottery satisfies the normalization condition

$$
\sum_{m=1}^{n} \frac{1}{2^{m}}+\frac{1}{2^{n}}=1
$$

Then the principal question "for how many tosses the player should buy a ticket" is equivalent to the question "which lottery can be treated as optimal in the sense of being preferred by the players?"

The above game is a well formulated mathematical problem and does not contain any paradox in its formulation.

\subsection{Original St. Petersburg paradox}

The paradox appears in the attempt to suggest a normative mathematically justified way of choosing an optimal lottery from the given set of lotteries in the Bernoulli game, so that the prescribed choice would be in agreement with the choice of real players.

In the original setup, one compares the lottery expected utilities. The lottery enjoying the largest expected utility is nominated optimal. One considers a linear utility function $u(x)=x$ of payoffs $x$. In general, one could use an affine utility function as $u(x)=$ const $+c x$. It is clear, however, that without loss of generality, the constant term can be omitted and the coefficient $c$ can be set to one by choosing the appropriate monetary units. The St. Petersburg paradox does not depend on these minor details. So, for the simplicity of notation, it is sufficient to use the linear function.

The lottery expected utility is commonly considered as the lottery value or the price of the ticket that is worth paying for entering the lottery. It is exactly this meaning that is kept in mind, when one talks of the lottery value in the St. Petersburg paradox. This value directly depends on the prize that one can win by playing the game represented by the lottery. Therefore, one usually considers as equivalent the terms lottery utility, lottery price, and lottery payoff.

Thus, using the rules of expected utility theory (von Neumann and Morgenstern (1944), Savage (1954)), one comes to the utility of lottery (1)

$$
U_{n}=\sum_{m=1}^{n} u\left(2^{m}\right) \frac{1}{2^{m}}=n
$$

As is evident, the largest expected utility corresponds to the lottery with the infinite number of tosses:

$$
U_{\text {max }}=U_{\infty} \quad(n \rightarrow \infty) .
$$

Since the largest expected utility, with infinite payoff, assuming the infinite number of tosses, by definition is optimal, one is led to conclude that any rational player should decide to always enter the game for any arbitrarily large price. However, in reality, real human players would not play for more than a few monetary units (Bernoulli (1738)), hence the paradox. 


\subsection{General formulation of Bernoulli game}

It is clear that the considered problem can be generalized to a set $\left\{L_{n}\right\}$ of the lotteries

$$
L_{n}=\left\{x_{1}, p_{1}\left|x_{2}, p_{2}\right| \ldots\left|x_{n}, p_{n}\right| 0, p_{n}\right\},
$$

with payoffs $x_{m}$ and their associated probabilities $p_{m}$. These probabilities can be either objective (von Neumann and Morgenstern (1944)) or subjective (Savage (1954)), as this has no impact on the nature of the problem. But, of course, the probability measure over the outcomes is required to be normalized,

$$
\sum_{m=1}^{n} p_{m}+p_{n}=1
$$

The question is: Which lottery from the set of lotteries $\left\{L_{n}: n=1,2, \ldots, \infty\right\}$ is optimal?

\subsection{General formulation of St. Petersburg paradox}

According to utility theory, the lottery with the largest expected utility

$$
U_{\text {max }}=\max _{n} U_{n}, \quad U_{n}=\sum_{m=1}^{n} u\left(x_{m}\right) p_{m},
$$

is accepted as optimal. However, it is easy to show (Seidl (2013)) the following.

Theorem 1. For each nonincreasing probability distribution $p_{m}$, there exist nondecreasing utility functions $u\left(x_{m}\right)$ such that the expected utility $U_{n}$ diverges as $n \rightarrow \infty$.

Proof. For each probability distribution $p_{m}$, it is possible to define such utility functions $u\left(x_{m}\right)$ that for all $m>m^{*}$ one has the inequality

$$
\frac{u\left(x_{m+1}\right) p_{m+1}}{u\left(x_{m}\right) p_{m}} \geq 1 \quad\left(m \geq m^{*}\right) .
$$

Then by the d'Alembert ratio test the sequence $\left\{U_{n}\right\}$ diverges as $n \rightarrow \infty$.

As an explicit example, let us take the linear utility function and the standard for the St. Petersburg paradox probability distribution $p_{m}=1 / 2^{m}$, then

$$
U_{n}=\frac{x\left(2^{n}-x^{n}\right)}{2^{n}(2-x)} \quad\left(u\left(x_{m}\right)=x^{m}, p_{m}=\frac{1}{2^{m}}\right) .
$$

In particular, for $x=2$ we have

$$
U_{n}=n \quad(x=2) .
$$

Therefore $U_{n}$ diverges, as $n \rightarrow \infty$, for $x \geq 2$ and converges for $x<2$.

If the optimal lottery, with the largest expected utility, corresponds to $n \rightarrow \infty$ and has the infinite value, then any rational player should feel it profitable to spend all available money to buy a ticket allowing for the maximal possible number of tosses. But numerous empirical data drastically contradict this conclusion, since the majority of real players prefer the lotteries with quite a modest number of tosses (Bottom et al. (1989), Rivero et al. (1990), Vivian (2004), Hayden and Platt (2009), Cox et al. (2009), Neugebauer (2010), Cox et al. (2018), Nobandegani and Shultz (2020)). This contradiction is the essence of the paradox. 


\section{Attempts of Paradox Solution}

The contradiction between the theoretical prediction of what should be the optimal lottery and the understanding of real human beings, appearing in the St. Petersburg paradox, looks very strange. Several attempts have been made to resolve the paradox. However all these attempts have not addressed the original paradox, but suggested changes to the original formulation by replacing it with some other problems. Such replacements cannot be considered as providing solutions of the original paradox (Clark (2002), Martin (2008)).

\subsection{Use concave utility functions}

The first suggestion was made by Cramer (1728) and Bernoulli (1738) who proposed to take a utility function characterized by a decreasing marginal utility, such as the square-root function (Cramer (1728)) or the logarithmic utility function $u(x)=\ln x$ (Bernoulli (1738)), leading to finite expected utilities. Taking, for instance, the logarithmic utility function gives

$$
\sum_{m=1}^{\infty} \frac{m \ln 2}{2^{m}}=2 \ln 2 .
$$

This approach opened the road towards the classical decision theory under uncertainty, in which risk aversion and decreasing marginal utility of gains are quantified by increasing concave utility functions.

However, this so-called "solution" does not solve the initial paradox, since the utility function has been modified, in the sense that it still accepts that a supposedly well-defined lottery with the linear utility function may deliver an infinite average gain. Furthermore, even if we accept the change of utility function, it is straightforward to recover a paradox of the same type by changing the prize structure. Suppose, for instance, that, instead of paying $x_{m}=2^{m}$ for a run of $m$ heads followed by tails, the prize is set to $x_{m}=\exp \left(2^{m}\right)$. Then, the expected utility, with the logarithmic utility function, becomes infinite again. As suggested by Menger (1967), it is possible to generalize this result and state that, for any utility function, there is a prize structure such that the expected utility diverges.

\subsection{Assume the boundness of utility functions}

Menger (1967) argued that the only way of resolving the paradox is to assume that there is an upper limit to utility. The same assumption was accepted by Hardin (1982), Jeffrey (1983), and Gustason (1994). In that case, the expected utility remains finite whatever the prize structure.

The finiteness of the utility function finds a justification in the neuroeconomics of sensation. There are arguments that, because of the finite rates of neuronal firing of action potentials, subjective values should be non-increasing beyond some point (Rayo and Becker (2007), Glimcher and Tymula (2018)). A similar saturation of sensation occurs for major disasters for which human moral intuitions are not well-calibrated to the extreme scales of deaths. This is called moral numbing (Slovic et al. (2020)).

Martin (2008) argued that limiting the utility function by some value is a quite subjective procedure. Such limits, if any, would be very different for different people and for different circumstances. They certainly cannot be applied to all people without exception with respect 
to money. There exist a number of examples when for people there is no limit of money utility, and the more they have, the better (Martin (2008)).

\subsection{Change the expected utility definition}

Generally, it is always possible to change the definition of the expected utility in order to obtain a finite value for the newly defined quantity. For instance, one can include some risk-aversion factors in the definition (Weirich (1984)).

There are however several objections to this approach. In fact, some people are not risk averse in certain situations and can even enjoy taking risk. It is also possible to adjust the prize structure in order to compensate the risk aversion and make again infinite the expected utility, as discussed by Martin (2008). Using cumulative prospect theory (Kahneman and Tversky (1979)), instead of expected utility theory, also does not remove the paradox, unless one artificially selects some special probability distributions and parameters (Rieger and Wang (2006)), which transforms the problem into a form having little relation to the original Bernoulli setup. Blavatskyy (2005) showed that the cumulative prospect theory cannot explain the paradox, since it requires to overweight small probabilities which more than offsets the concavity of wealth. In any case, changing the rules of the game is not a solution to the original problem. As Pfiffermann (2011) concludes "Cumulative prospect theory does not explain the St. Petersburg paradox".

\subsection{Set a finite limit to prizes}

Since no individual and no casino possess infinite resources, the total prize should be limited by a finite value (Samuelson (1960), Gustason (1994)). This means that the summation in $U_{\infty}$ should be cut at a finite $N$ by assuming that, either the utility function or the probabilities take zero values for all $n>N$.

Mathematically, this corresponds to changing the original Bernoulli game, by either a different definition of the utility function or of the payoffs, or of the probabilities. Therefore, it cannot be considered to be a solution to the initial problem.

\subsection{Set a finite number of tosses}

Many argued (see, e.g., Nathan (1984) and references therein) that, in reality, nobody can toss a coin an infinite number of times, so that the summation in $U_{\infty}$ must be limited by a finite $N$.

Mathematically, this is just a particular case of the same modification of the game as described in the previous variant. Therefore, the original problem remains open. As Martin (2008) says, "refusing to think about the problem is not solving it".

\subsection{Replace the single game by a set of repeated games}

Sometimes one replaces the single Bernoulli game described above by a set of repeated games played $N$ times, as is described by Vivian (Vivian (2013)). The set of $N$ repeated games can be treated as a compound lottery characterized by an expected monetary value $U_{N}$ defined as the average per game monetary value. As has been found (Vivian (2013)), this expected value 
is $U_{N}=1+\log _{2} N$. Although $U_{N}$ increases to infinity with $N \rightarrow \infty$, but this increase is much slower than the rise of $U_{n}=n$ with $n \rightarrow \infty$ in the original Bernoulli game. Experimental data confirm this behaviour (Klyve and Lauren (2011), Vivian (2013)).

However, replacing one game by another is not the solution of the problem. Moreover, from the mathematical point of view the same problem remains. In the original Bernoulli game, one is given a set of alternatives valued as $U_{n}=1,2, \ldots, \infty$, and the question is posed of deciding which of the alternatives is preferable. If the criterion of preference is the maximal $U_{n}$, then the choice is $U_{\infty}$. But the willingness to pay is finite and not large.

In the same way, if one is given a collection of expected values $U_{N}=1+\log _{2} N$, with $N=1,2, \ldots, \infty$, and the choice is prescribed by the largest $U_{N}$, then this choice is again $U_{\infty}$, while the willingness to pay is finite. As earlier, the problem is not in the formulation of the game, but in the contradiction between the largest expected value and the willingness to pay. A detailed consideration is given in Sec. 9.

\subsection{Conclusion on the attempted "solutions"}

By changing the rules of the Bernoulli game, it is certainly possible to adjust them so that the expected utility or an equivalent decision functional be finite. All previously suggested "solutions" are exactly of this type. One solves some variants that are not the original problem. In that sense, many versions could be invented to replace the original St. Petersburg paradox (Samuelson (1977)). However, "imposing restrictions on theory to rule out St. Petersburg bath water would throw out some babies as well" (Martin (2008)). The commonly accepted conclusion is that "from the mathematical and logical point of view, the St. Petersburg paradox is impeccable" (Resnik (1987)). The suggested "solutions" are not mathematical (Seidle (2013)). That is, the original St. Petersburg paradox remains unresolved.

\section{Brief Account on Stochastic Decision Making}

The resolution of the St. Petersburg paradox, advanced in the present paper, is based on a probabilistic approach in decision theory. Therefore it is necessary to mention, at least briefly, the main models of this approach and to emphasize the basic points justifying it.

Generally speaking, in decision theory there are several approaches involving probabilistic choice. The simplest model is a tremble model of Harless and Camerer (1994). According to this model, individuals have a unique preference relation on the set of lotteries, but they do not choose following their preferences all the time. Instead, because of a random involutionary tremble occurring with some probability $p$ individuals choose an alternative which is not their preferred option. Individuals act in accordance with their preferences with probability $1-p$. However, Carbone (1997) and Loomes et al. (2002) find that this tremble model fails to explain experimental data.

The other is the Fechner (1860) model of random errors where individuals possess a unique preference relation on the set of lotteries, but they reveal their preferences with a random error as a result of carelessness, slips, insufficient motivation etc. In the Fechner model, random error is usually drawn from a normal distribution with zero mean and constant standard deviation (Hey and Orme (1994)) or from a truncated normal distribution (Blavatskyy (2006)). Under 
the Fechner model, errors are more likely when the utilities of the options are close and less likely when they are less close. That is not the same as in the tremble model, where every choice over lottery pairs has the same error rate.

The third model of stochastic choice is a random preference model, where it is assumed that individuals are endowed with several preference relations over the set of risky lotteries and a probability measure over these preference relations. When faced with a decision problem, individuals first draw a preference relation and then choose an alternative which they prefer according to the selected preference relation (Loomes and Sugden (1995)).

Finally, in the random choice model one assumes that individuals possess a probability measure that captures the likelihood of a lottery being chosen over other lotteries. Then there is no need for a mediator between a deterministic preference relation and an empirical stochastic choice pattern. The lotteries $L_{n}$ and, respectively, the related expected utilities are considered as random quantities characterized by a probability distribution $p\left(L_{n}\right)$ over the lotteries (Luce (1959, 1977), Gul et al. (2014)). One should not confuse the probabilities over the set of lotteries with the probabilities of payoffs inside each lottery.

The probabilistic approach we employ in the present paper is based on the ideas of the random choice model, however with an important generalization. The probability distribution over the set of lotteries is not just postulated but it is defined by the principle of minimal information taken in the form of the Kullback-Leibler information functional (Kullback and Leibler (1951), Kullback (1959)), where the Luce rule plays the role of a trial prior. The method of deriving probability distributions from the minimization of an information functional, or from the minimization of a conditional relative entropy under given constraints, is well known and widely used. The most mathematically grounded approach is based on the Shore-Jonson theorem (Shore and Jonson (1980)) stating that, given a prior density and new constraints, there is only one posterior density satisfying these constraints and the conditions of uniqueness, coordinate invariance, and system independence, such that this unique posterior can be obtained by minimizing the Kullback-Leibler information functional (Kullback and Leibler (1951), Kullback (1959)).

Numerous empirical studies demonstrate that practically all decision makers do not follow the absolutely deterministic choice between the given alternatives, but the decisions vary for different subjects and even for the same subject at different moments of time (Luce (1958, 1959, 1977), French and Insua (2000), Gul et al. (2014)).

The most important is that stochasticity in decisions is unavoidable even for a single decision maker in the process of each decision. This is due to the fact that, as has been discovered in neurophysiological studies, the decision process in the brain is accompanied by noise leading to random choice, with the randomness caused by generic variability and local instability of neural networks (Werner and Mountcastle (1963), Arieli et al. (1986), Gold and Shadlen (2001), Glimcher (2005), Schumacher et al. (2011), Shadlen and Shohamy (2016), Webb, R. (2019), Kurtz-David et al. (2019)). Neurophysiological studies provide evidence that the process of decision making in regions of the human brain that are known to be responsible for value estimation is highly variable. This variability causes choice inconsistency at repeated decisions. The variability arises not merely from limitations in the data available to the decision maker, but the choice itself is stochastic due to fundamental stochasticity in the physiological process of making decisions (Werner and Mountcastle (1963), Arieli et al. (1986), Gold and Shadlen (2001), Glimcher (2005), Schumacher et al. (2011), Shadlen and Shohamy (2016), Webb, R. 
(2019), Kurtz-David et al. (2019)).

In the recent review, Woodford (2020) summarizes the modern point of view that considers randomness as an internal feature of functioning of the human brain, where decisions are formed on the basis of noisy internal representation. The cognitive process through which judgments are generated involves two stages: encoding of the stimulus, that is the process through which the internal representation is produced, and decoding of the internal representation about the stimulus, both these stages are random, being accompanied by noise. The existence of random noise is caused by the way that neurons fire in response to the signals they receive.

In this way, psychophysical and neurophysiological studies evidence that cognitive imprecision is due to the inevitable internal noise. This noise in the nervous system corrupts the evidence that must subsequently be decoded to produce a judgment. Stochasticity is the unavoidable feature of the human brain functioning. As a result, the choice in decision making is not deterministic, being based on the comparison of utilities, but it is rather stochastic and based on the comparison of probabilities. It is important to stress that stochasticity is an internal property of a subject and it is always involved in the process of decision making at both the stages of encoding external signals and decoding them for formulating a decision. The internal stochastic fluctuations in the brain, which can be connected with the characteristics of external signals, are revealed in decision making as hesitations.

In the case of a subject deciding to take part in the Bernoulli gamble, hesitations can arise with respect to the rules of the gamble. First of all, a subject can have doubt whether he/she has correctly understood the gamble description. The possibility of gaining an enormous, or even infinite, payoff should certainly be suspicious for any reasonable person. A normal human, when assuming the possibility of an infinite gain would for sure feel that there is a kind of cheating here. Moreover, average humans have some limit of imagination with respect to the amount of money, above which numbers sound as purely fantastic. Such suspicions should inevitably induce strong disbelief with respect to the gamble. In turn, this disbelief would amplify the naturally existing stochasticity in the internal encoding-decoding process in the brain.

This is why in order to understand the origin of the St. Petersburg paradox, it is necessary to resort to the probabilistic approach in decision making. The Bernoulli game as such is absolutely correct and does contain an infinite lottery with infinite expected utility. However, for real humans the optimality of a lottery is not prescribed by its largest expected utility, but it is defined by the largest probability of the lottery. So there is no need to change anything in the Bernoulli game that is mathematically precise. The paradox is not in the game, but in the application to the game of the deterministic preference criterion. Therefore the paradox can be eliminated only by using the probabilistic preference criterion.

\section{Formulation of the Approach}

Suppose that a set of alternatives is presented as a set of lotteries $\left\{L_{n}: n=1,2, \ldots\right\}$. For the sequence $\left\{L_{n}: n=1,2, \ldots\right\}$ of these lotteries, there corresponds the sequence $\left\{U_{n} \equiv U\left(L_{n}\right)\right.$ : $n=1,2, \ldots\}$ of their expected utilities. The notion of the infinite lottery is defined as the limit of the sequence $\left\{L_{n}\right\}$ for $n$ tending to infinity, $n \rightarrow \infty$. According to the random choice approach, the lotteries are treated as random variables, with a probability measure $\left\{p\left(L_{n}\right): n=1,2, \ldots\right\}$ over the set of these lotteries. In other words, the choice is not deterministic, being based on the 
comparison of utilities, but it is rather stochastic and based on the comparison of probabilities. Below we formulate these properties explicitly. The use of stochastic preferences is the basic point of the approach based on the following definitions.

Definition 1. The lottery $L_{i}$ is stochastically preferred to $L_{j}$ if and only if

$$
p\left(L_{i}\right)>p\left(L_{j}\right) \quad\left(L_{i} \succ L_{j}\right) .
$$

Definition 2. The lotteries $L_{i}$ and $L_{j}$ are stochastically indifferent if and only if

$$
p\left(L_{i}\right)=p\left(L_{j}\right) \quad\left(L_{i} \sim L_{j}\right)
$$

Definition 3. The lottery $L_{n_{o p t}}$ is called stochastically optimal if and only if

$$
p\left(L_{n_{\text {opt }}}\right)=\sup _{n} p\left(L_{n}\right) .
$$

A stochastically optimal lottery corresponds to the maximal lottery probability. This implies that, according to the frequentist interpretation of probability, the majority of subjects choose exactly the stochastically optimal lottery where the probability possesses a maximum.

It is possible to study general properties of the probability measure and the process of stochastic optimization (Rubinstein and Kroese (2004), Gul et al. (2014)). However we concentrate our attention on the practical way of constructing an explicit expression for the probability, since its explicit formula is compulsory for the resolution of the St. Petersburg paradox.

The probability measure over the set of lotteries has to satisfy the necessary conditions (Gul et al. (2014), Yukalov and Sornette $(2014,2018)$ ) characterizing the sought probability. The first condition is the standard normalization for the probability

$$
\sum_{n=1}^{\infty} p\left(L_{n}\right)=1, \quad 0 \leq p\left(L_{n}\right) \leq 1 .
$$

The second condition is the existence of a global mean

$$
\sum_{n=1}^{\infty} p\left(L_{n}\right) U_{n}=U, \quad(|U|<\infty) .
$$

One should not confuse the probability $p_{n}$ of payoffs $x_{n}$ in a lottery with the probability of a lottery $p\left(L_{n}\right)$ as a whole. Respectively, one should not confuse the expected utility $U_{n}$ of a lottery $L_{n}$ with the global mean $U$ that is not an expected lottery utility, but just a constraint. The explicit form of the probability distribution $p\left(L_{n}\right)$ can be derived from the minimization of the Kullback-Leibler information functional (Kullback and Leibler (1951), Kullback (1959), Shore and Jonson (1980)). The Shore-Jonson theorem (Shore and Jonson (1980)) states that, given a prior density and additional constraints, there is only one posterior density satisfying these constraints and the conditions of uniqueness, coordinate invariance, and system independence, such that this unique posterior can be obtained by minimizing the Kullback-Leibler information functional (Kullback and Leibler (1951), Kullback (1959)). 
It is important to stress the following pivotal point. If one postulates the explicit form of the Kullback-Leibler information functional and defines the probability $p\left(L_{n}\right)$ as its minimizer, then this makes a sufficient condition for deriving the expression of $p\left(L_{n}\right)$, without requiring the finiteness of $U$ in constraint (13). However the Shore-Jonson theorem (Shore and Jonson (1980)) is more general. In addition to the above sufficient condition, it states that, under given constraints and conditions of uniqueness, coordinate invariance, and system independence, the posterior probability $p\left(L_{n}\right)$ is necessarily the minimizer of a functional enjoying the Kullback-Leibler form, provided the imposed constraints, such as (12) and (13) do not contain singularities, which here means a not divergent value of $U$. The Shore-Jonson theorem justifies why it is precisely the Kullback-Leibler functional that has to be accepted for the minimization procedure.

In the present case, this information functional for the posterior probability distribution $p\left(L_{n}\right)$, under a prior distribution $p_{0}\left(L_{n}\right)$ and constraints (12) and (13), reads as

$$
I[p]=\sum_{n=1}^{\infty} p\left(L_{n}\right) \ln \frac{p\left(L_{n}\right)}{p_{0}\left(L_{n}\right)}+\alpha\left[1-\sum_{n=1}^{\infty} p\left(L_{n}\right)\right]+\beta\left[U-\sum_{n=1}^{\infty} p\left(L_{n}\right) U_{n}\right],
$$

where $\alpha$ and $\beta$ are Lagrange multipliers. The Kullback-Leibler information functional is a measure for the amount of information necessary to transform a prior probability distribution $p_{0}\left(L_{n}\right)$ into the posterior distribution $p\left(L_{n}\right)$. The choice of a prior distribution will be explained below.

The minimization of the Kullback-Leibler information functional is equivalent to the minimization of the Kullback-Leibler relative entropy, or divergence, under given constraints. This concept is well known and widely employed in statistical models of inference, different branches of applied statistics, information theory, computing, machine learning, pattern recognition, development of artificial intelligence, mechanics, neuroscience, theory of biological systems, and various kinds of optimization problems (Kullback and Leibler (1951), Kullback (1959), Burnham and Anderson (2002), MacKay (2003), Nielsen (2005), Cover and Thomas (2006)). As is explained above, the Shore-Jonson theorem (Shore and Jonson (1980)) justifies why the posterior distribution has to be defined as the minimizer of the Kullback-Leibler functional. When minimizing this functional, one can meet a finite index set $n=1,2, \ldots, N$ as well as the infinite index set $n=1,2, \ldots, \infty$. The rigorous procedure of the minimization is done for a finite index set, with a finite $N$, and the transition to an infinite index set $n=1,2, \ldots, \infty$ is accomplished after the minimization procedure by sending $N \rightarrow \infty$. The details of the transition $N \rightarrow \infty$ can be found in literature cited above and in (Dmitruk and Kuzkina (2005), Pinski (2015)). The minimization theorem for a finite $N$ is formulated below.

Theorem 2. The probability distribution $p\left(L_{n}\right): n=1,2, \ldots, N$, defined as the minimizer of the Kullback-Leibler information functional

$$
I_{N}[p]=\sum_{i=1}^{N} p\left(L_{n}\right) \ln \frac{p\left(L_{n}\right)}{p_{0}\left(L_{n}\right)}+\alpha\left[1-\sum_{n=1}^{N} p\left(L_{n}\right)\right]+\beta\left[U-\sum_{n=1}^{N} p\left(L_{n}\right) U_{n}\right]
$$

reads as

$$
p\left(L_{n}\right)=\frac{p_{0}\left(L_{n}\right) e^{\beta U_{n}}}{\sum_{n=1}^{\infty} p_{0}\left(L_{n}\right) e^{\beta U_{n}}} .
$$


Proof. The proof is based on the procedure of minimizing functionals in calculus of variations (Gelfand and Fomin (1963)). The necessary condition for the functional $I[p]$ to be an extremum is the zero first-order variation

$$
\delta I[p]=\sum_{n} \frac{\partial I[p]}{\partial p\left(L_{n}\right)} \delta p\left(L_{n}\right)=0 .
$$

From here, using the Kullback-Leibler information functional, we have

$$
\frac{\partial I[p]}{\partial p\left(L_{n}\right)}=\ln \frac{p\left(L_{n}\right)}{p_{0}\left(L_{n}\right)}+1-\alpha-\beta U_{n}=0,
$$

which, together with the normalization condition, yields expression (15).

The necessary condition for the extremum to be a minimum is the non-negativity of the second-order variation. This condition becomes also sufficient if the second-order variation is strictly positive,

$$
\delta^{2} I[p]=\frac{1}{2} \sum_{m n} I_{m n} \delta p\left(L_{m}\right) \delta p\left(L_{n}\right)>0
$$

where

$$
I_{m n}=\frac{\partial^{2} I[p]}{\partial p\left(L_{m}\right) \partial p\left(L_{n}\right)} .
$$

The second-order variation is positive provided that the Hessian matrix $\left[I_{m n}\right]$, composed of the elements $I_{m n}$, is positive, which requires that all principal minors of this Hessian matrix be positive. It is easy to see that the Hessian matrix is diagonal, since

$$
I_{m n}=\frac{\delta_{m n}}{p\left(L_{n}\right)} .
$$

Therefore for its positivity, it is necessary and sufficient that

$$
I_{n n}=\frac{\partial^{2} I[p]}{\partial p\left(L_{n}\right)^{2}}=\frac{1}{p\left(L_{n}\right)}>0,
$$

that is the probability $p\left(L_{n}\right)$ has to be positive.

Remark. According to the standard arguments (Burnham and Anderson (2002), MacKay (2003), Nielsen (2005), Cover and Thomas (2006), Dmitruk and Kuzkina (2005), Pinski (2015)), after the minimization procedure, the finite index set can be extended to infinite by sending $N$ to $\infty$.

As a trial prior, it is possible to take the Luce $(1958,1959,1977)$ form defined as follows. If the considered alternatives are characterized by the related attributes $a_{n} \geq 0$, then the trial probability of choosing an alternative $L_{n}$ is

$$
p_{0}\left(L_{n}\right)=\frac{a_{n}}{\sum_{n=1}^{\infty} a_{n}} .
$$

Hence the posterior distribution (15) becomes

$$
p\left(L_{n}\right)=\frac{a_{n} e^{\beta U_{n}}}{\sum_{n=1}^{\infty} a_{n} e^{\beta U_{n}}} .
$$


The first and direct attribute of a lottery is given by its expected utility. Therefore, for the case of semi-positive expected utilities, the latter can be accepted as the lottery attributes, while for negative expected utilities, the lottery attributes can be defined as inverse lottery absolute values,

$$
a_{n}=\left\{\begin{array}{cc}
U_{n}, & U_{n} \geq 0 \\
\left|U_{n}\right|^{-1}, & U_{n}<0
\end{array} .\right.
$$

Thus we obtain the explicit expressions for the probability distribution.

Definition 4. The probability distribution over the set of lotteries with semi-positive expected utilities has the form

$$
p\left(L_{n}\right)=\frac{U_{n} e^{\beta U_{n}}}{\sum_{n=1}^{\infty} U_{n} e^{\beta U_{n}}} \quad\left(U_{n} \geq 0\right),
$$

and for the lotteries with negative expected utilities,

$$
p\left(L_{n}\right)=\frac{\left|U_{n}\right|^{-1} e^{\beta U_{n}}}{\sum_{n=1}^{\infty}\left|U_{n}\right|^{-1} e^{\beta U_{n}}} \quad\left(U_{n}<0\right) .
$$

It is useful to note that these distributions differ from the logit form, which is often postulated. As is seen, the lottery probability $p\left(L_{n}\right)$ depends on the expected utility $U_{n}$ of this lottery. According to the criterion of stochastic preference, the optimal lottery corresponds to the maximal lottery probability.

The parameter $\beta$, from the mathematical point of view, is a Lagrange multiplier guaranteeing the normalization condition (13). From the psychological side, the parameter $\beta$ plays the role of a belief parameter reflecting the belief of a subject in the fairness of the gamble and in the subject confidence with respect to his/her understanding of the overall rules and conditions of the gamble. Under neutral belief, when $\beta=0$, the probability distribution returns to the Luce form (16). In the case of strong belief, the situation reduces to the deterministic choice of the largest expected utility:

$$
p\left(L_{n}\right)=\left\{\begin{array}{ll}
1, & U_{n}=U_{\max } \\
0, & U_{n} \neq U_{\max }
\end{array} \quad(\beta \rightarrow \infty),\right.
$$

where

$$
U_{\max } \equiv \max _{n} U_{n}
$$

It also may happen strong disbelief, when $\beta \rightarrow-\infty$, and it becomes more profitable to choose the lottery with the minimal expected utility. Although in realistic cases, the belief parameter is finite. It can be positive in the case of belief or negative in the case of disbelief.

When all expected utilities are finite, that is $\left|U_{n}\right|<\infty$, then the probability distribution satisfies all normalization conditions by construction, including the normalization condition (13). The situation is more restrictive, when the maximal expected utility (22) diverges, so that $U_{\max } \rightarrow \infty$, as it happens in the St. Petersburg paradox.

Theorem 3. If the maximal expected utility (22) diverges, so that $U_{\max } \rightarrow \infty$, then the normalization condition (13) requires that $\beta$ be negative. 
Proof. From the expression for the probability distribution (19) or (20), it follows that

$$
p\left(L_{\max }\right)=\left\{\begin{array}{ll}
1, & \beta \geq 0 \\
0, & \beta<0
\end{array} \quad\left(U_{\max } \rightarrow \infty\right) .\right.
$$

If $\beta$ is semi-positive and $U_{\max } \rightarrow \infty$, then the normalization condition (13) does not hold, since $U \rightarrow U_{\max } \rightarrow \infty$. This condition (13) is satisfied only for $\beta<0$.

Remark. Similarly, it is easy to show that if the minimal expected utility tends to $-\infty$, then $\beta$ must be semi-positive. In the case of the Bernoulli game, the parameter $\beta$ has to be negative, since the expected utilities are positive defined and can reach infinite value. A negative parameter $\beta$ signifies disbelief that can be caused e.g. by the disbelief of decision makers in the fairness of the game or by their disbelief in the principal possibility of gaining an infinite sum.

\section{A Resolution of St. Petersburg Paradox}

Now we are in a position to formulate a resolution of the St. Petersburg paradox. Recall that to resolve the paradox, it is necessary to explain, why in the Bernoulli game, despite a divergent maximal expected utility, people consider as optimal a lottery with a finite expected utility, and respectively, with a finite number of tosses.

Theorem 4. Assume that in the Bernoulli game the maximal expected utility diverges. Then, under the probability distribution (19), the stochastically optimal lottery has a finite expected utility corresponding to a finite number of tosses.

Proof. In the Bernoulli game, the expected utility of a lottery with $n$ tosses is positive, $U_{n}>0$. By assumption, the largest expected utility diverges, $U_{\max } \rightarrow \infty$, hence the belief parameter, according to Theorem 3 , has to be negative, $\beta<0$, corresponding to disbelief. Therefore the probability distribution over lotteries has the form

$$
p\left(L_{n}\right)=\frac{U_{n}}{Z} e^{\beta U_{n}},
$$

with the normalization factor

$$
Z=\sum_{n=1}^{\infty} U_{n} e^{\beta U_{n}} \quad(\beta<0) .
$$

By Definition 3 in equation (11), the stochastically optimal lottery $L_{n_{o p t}}$ is defined by the maximum of the probability $p\left(L_{n}\right)$, where

$$
\frac{\partial p\left(L_{n}\right)}{\partial U_{n}}=0 \quad\left(L_{n}=L_{n_{o p t}}\right) .
$$

Then the stochastically optimal lottery corresponds to the expected utility

$$
U_{o p t}=U\left(L_{n_{o p t}}\right)=\frac{1}{|\beta|} .
$$


This utility is finite for any negative value of the belief parameter, and there is no paradox.

For illustration, let us consider the original Bernoulli game, where $U_{n}=n$. This leads to the probability

$$
p\left(L_{n}\right)=\frac{n}{Z} e^{\beta n} \quad(\beta<0),
$$

with the normalization factor

$$
Z=\sum_{n=1}^{\infty} n e^{-|\beta| n}=\frac{1}{4 \sinh ^{2}(|\beta| / 2)} .
$$

In the optimal lottery, the number of tosses is $n_{\text {opt }} \approx 1 /|\beta|$. Keeping in mind that $n_{\text {opt }}$ is to be a whole number, it lays in the interval

$$
\left[\frac{1}{|\beta|}\right] \leq n_{\text {opt }} \leq\left[\frac{1}{|\beta|}\right]+1
$$

where the square brackets imply the entier (the greatest integer not exceeding the specified number).

Empirical investigations of the Bernoulli game (Cox et al. (2009, 2018), Hayden and Platt (2009), Neugebauer (2010), Nobandegani and Shultz (2020)) show that the maximal fraction of players prefers one or two steps in the game. Keeping in mind the frequentist understanding of probability implies that the maximal number of players considers as stochastically optimal the game of one - two tosses, that is $n_{\text {opt }} \approx 1,2$. Therefore, according to condition (28), we have $|\beta| \approx 1$. Thus, despite that the maximal expected utility of the Bernoulli toss game, is infinite, the majority of real human beings would not evaluate it so much, but, in contrast, would not buy a ticket for more than a few monetary units.

\section{Behavioral Meaning of Belief Parameter}

Mathematically, the parameter $\beta$ appears as a Lagrange multiplier in the process of the Kullback-Leibler information-functional minimization. Its interpretation as of a parameter characterizing the belief (or disbelief) of a decision maker is based on its role. It is termed belief parameter when $\beta>0$ and disbelief parameter if $\beta<0$. As is shown in Sec. 5, in the case of strong belief, when $\beta \rightarrow \infty$, we return to the deterministic variant of decision theory prescribing that $100 \%$ of decision makers should prefer the lottery with the largest expected utility. In the opposite case of strong disbelief, when $\beta \rightarrow-\infty$, one chooses the lottery with the minimal expected utility. And $\beta=0$ characterizes the neutral situation, when there is neither noticeable belief nor disbelief.

In the considered case of the St. Petersburg paradox, the behavioral meaning of the belief parameter is justified by the attitude of a decision maker to the game. The formulation of the Bernoulli gamble suggests a seeming possibility of gaining infinite (or at least enormous) amount of money. For usual people, such a possibility sounds as highly unrealistic, because of which they do not give much trust to this promise. That is, a normal person does not believe in the fairness of the gamble. This is why the belief parameter is negative for this case.

As is evident, the value of the belief parameter is closely connected with the particular game. In the Bernoulli game, from the behavioural point of view, there is disbelief in the 
fantastically sounding aptitude of winning infinite amount of money, because of which this parameter is negative. Being a Lagrange multiplier, the value of this parameter, as is usual for optimization problems, can be found from the knowledge of some phenomenological data or from additional constraints. For example, the value of the disbelief can be connected with the level of uncertainty in the game. For a set of lotteries, the typical quantity characterizing the level of uncertainty is the standard deviation (Ghahramani (2000)). Considering a set of lotteries $L_{n}$ with the expected utilities $U_{n}$ and probabilities $p\left(L_{n}\right)$, the standard deviation is

$$
\sigma=\sqrt{\operatorname{var}\left(U_{n}\right)},
$$

which is a square root of the variance

$$
\operatorname{var}\left(U_{n}\right)=\left\langle U_{n}^{2}\right\rangle-\left\langle U_{n}\right\rangle^{2},
$$

where

$$
\left\langle U_{n}\right\rangle=U=\sum_{n} U_{n} p\left(L_{n}\right), \quad\left\langle U_{n}^{2}\right\rangle=\sum_{n} U_{n}^{2} p\left(L_{n}\right) .
$$

The larger the standard deviation, the larger the uncertainty in the choice among the alternatives, hence the larger the absolute value of the disbelief represented by the parameter $\beta$. This allows us to consider the disbelief parameter being proportional to the standard deviation,

$$
\beta=-|\beta|=-\sigma \quad(\beta<0) .
$$

For the original formulation of the Bernoulli game, we have the expected utilities $U_{n}=n$, with the probabilities (27). Therefore we have

$$
\begin{gathered}
\left\langle U_{n}\right\rangle=U=\sum_{n=1}^{\infty} n p\left(L_{n}\right)=\operatorname{coth}\left(\frac{|\beta|}{2}\right), \\
\left\langle U_{n}^{2}\right\rangle=\sum_{n=1}^{\infty} n^{2} p\left(L_{n}\right)=\frac{1}{2}\left(3 U^{2}-1\right) .
\end{gathered}
$$

Then the variance (30) becomes

$$
\operatorname{var}\left(U_{n}\right)=\frac{1}{2}\left(U^{2}-1\right)=\frac{1}{2 \sinh ^{2}(|\beta| / 2)} .
$$

In this way, for the disbelief parameter (32) we obtain the equation

$$
\sqrt{2}|\beta| \sinh \frac{|\beta|}{2}=1,
$$

whose solution gives $|\beta|=1.157$. This estimate, according to the previous section, tells us that the optimal lottery corresponds to $n_{\text {opt }}$ of order one, that is, to the game of just a few tosses. This is in perfect agreement with the empirical observations that the majority of subjects prefer the Bernoulli game of one - two steps (Cox et al. (2009, 2018), Hayden and Platt (2009), Neugebauer (2010), Nobandegani and Shultz (2020)). 


\section{Role of Prior Probability}

The form of the lottery probability $p\left(L_{n}\right)$ that is the minimizer of the information functional, generally, depends of the prior probability $p_{0}\left(L_{n}\right)$. The latter was chosen above in the simple form satisfying the Luce rule. This simple form has the advantage of containing no additional parameters complicating the consideration. However, it is possible to put the question: What are the general conditions imposed on the prior probability, which allow for the solution of the St. Petersburg paradox?

It is evident that the prior probability distribution, by its meaning, cannot be represented by an arbitrary distribution, but has to satisfy reasonable assumptions appropriately describing the expected features of the problem. Thus, it is reasonable to assume that the prior probability, in addition to being semi-positive, should depend on the lottery expected utility $U_{n}$ so that, the increasing expected utility $U_{n}$ would increase $p_{0}\left(L_{n}\right)$, which means that

$$
\frac{\partial p_{0}\left(L_{n}\right)}{\partial U_{n}}>0 .
$$

Without the loss of generality, the prior probability can be written as

$$
p_{0}\left(L_{n}\right)=\frac{\varphi\left(U_{n}\right)}{\sum_{n} \varphi\left(U_{n}\right)} .
$$

The function $\varphi$, in view of condition (36), has the property

$$
\frac{\partial \varphi\left(U_{n}\right)}{\partial U_{n}}>0 .
$$

Then the minimizer of the information functional takes the form

$$
p\left(L_{n}\right)=\frac{\varphi\left(U_{n}\right) e^{\beta U_{n}}}{\sum_{n} \varphi\left(U_{n}\right) e^{\beta U_{n}}} .
$$

The sufficient conditions for the existence of a finite stochastically preferred lottery, are the conditions of the probability maximum

$$
\frac{\partial p\left(L_{n}\right)}{\partial U_{n}}=0, \quad \frac{\partial^{2} p\left(L_{n}\right)}{\partial U_{n}^{2}}<0 \quad\left(U_{n}=U_{n_{\text {opt }}}\right) .
$$

By using the notation

$$
x \equiv U_{n}, \quad x_{o p t} \equiv U_{n_{o p t}},
$$

these conditions can be rewritten as

$$
\varphi^{\prime}(x)+\beta \varphi(x)=0, \quad \varphi^{\prime \prime}(x)-\beta^{2} \varphi(x)<0 \quad\left(x=x_{\text {opt }}\right),
$$

where the primes imply the derivatives over $x$.

In this way, any function $\varphi$ satisfying conditions (38) and (42) will lead to the same general conclusion of the existence of a finite stochastically preferred lottery, that is, to the resolution of 
the paradox. Concrete formulas defining the optimal lottery will, of course, be slightly different. For instance, a simple function, satisfying condition (38), could be

$$
\varphi(x)=x^{\alpha} \quad(\alpha>0) .
$$

Then the first of conditions (42) gives

$$
x_{o p t}=\frac{\alpha}{|\beta|}
$$

while the second of conditions (42) is valid for any $\alpha>0$.

Another example is a logarithmic function $\varphi$, such that

$$
\varphi(x)=\ln (1+x)
$$

with the notation

$$
x \equiv \frac{U_{n}}{U_{0}}, \quad x_{o p t} \equiv \frac{U_{n_{o p t}}}{U_{0}},
$$

where $U_{0}>0$ is a scaling constant. Then the optimal lottery is defined by the equation

$$
\left(1+x_{o p t}\right) \ln \left(1+x_{o p t}\right)=\frac{1}{|\beta|}
$$

the second of conditions (42) being always valid.

One more example can be based on the general logit model that is widely employed in economics and stochastic decision theory. In the general version of the logit model, the trial probability of an alternative $L_{n}$ is assumed to have the form

$$
p_{0}\left(L_{n}\right)=\frac{e^{V_{n}}}{\sum_{n} e^{V_{n}}},
$$

where $V_{n}$ is the expected value of $L_{n}$. The expression for the value $V_{n}$ is to be postulated and might be very different from expected utility, e.g. as in the stochastic approach based on prospect theory (Kahneman and Tversky (1979)). In the majority of cases, the expected value $V_{n}$ is defined as a transformation $V_{n}=V\left(U_{n}\right)$ of the expected utility $U_{n}$. There exists a large variety of such transformations, including highly nonlinear ones (Small (1987), Gerken (1991), Wen and Koppelman (2001), Williams (2016)). For the illustration of the idea, let us take a weakly nonlinear transformation representing the value $V_{n}$ in the form

$$
V_{n}=V\left(U_{n}\right)=b_{n} U_{n}^{\gamma_{n}}+c_{n}
$$

where $b_{n}, c_{n}$, and $\gamma_{n}$ are positive parameters. These parameters are usually fitted to a particular problem of interest. As in the case of the St. Petersburg paradox, the expected utility is assumed to be in the interval $U_{n} \in[0, \infty)$ and the belief parameter $\beta$ is negative, expressing disbelief. The probability distribution (15) becomes

$$
p\left(L_{n}\right)=\frac{\exp \left(V_{n}+\beta U_{n}\right)}{\sum_{n} \exp \left(V_{n}+\beta U_{n}\right)} .
$$


The stochastically preferable alternative corresponds to the maximum of this probability, which gives the alternative with the expected utility

$$
U_{n_{o p t}}=\left(\frac{b_{n} \gamma_{n}}{|\beta|}\right)^{1 /\left(1-\gamma_{n}\right)},
$$

where $0<\gamma_{n}<1$.

In the general case of a transformation $V_{n}=V\left(U_{n}\right)$, the stochastically preferable alternative is defined by the equations

$$
\beta+\frac{\partial V_{n}}{\partial U_{n}}=0, \quad \frac{\partial^{2} V_{n}}{\partial U_{n}^{2}}<0 .
$$

Thus, there is a wide class of prior probabilities, satisfying straightforward conditions, which lead to the existence of a finite stochastically preferred lottery, hence resolving the paradox. It is worth emphasizing that this does not impose any constraints on the Bernoulli game as such, which does contain the limiting lottery with infinite expected utility. However the latter is not stochastically preferred.

\section{Repeated Games}

In the above consideration, we were keeping in mind a set of single Bernoulli games, consisting of $n=1,2, \ldots$ tosses corresponding to the lotteries $L_{n}$ with the expected utilities $U_{n}$. The aim was to find an optimal lottery $L_{n_{o p t}}$ that would be preferable. It is necessary to distinguish the expected utility or its particular form called the expected value, or the expected monetary value, and the willingness to pay. The expected value $U_{n}$ can increase to infinity with rising $n$, but the willingness to pay, that is denoted as $U_{n_{\text {opt }}}$, is finite. The mathematical formulation of the Bernoulli game is absolutely accurate and contains no paradoxes. Under the paradox, one assumes the apparent difference between the maximal expected value $U_{\max }=\max _{n} U_{n}$ and the willingness to pay $U_{n_{\text {opt }}}$. In the probabilistic approach above, it is explained that these notions are really different and do not need to coincide, thus removing the seeming paradox.

The same consideration is applicable to other games. For example, it is possible to consider a set of compound Bernoulli games, of $k$ tosses each, consisting of subsets of Bernoulli games repeated $N=2^{k}$ times (Tversky and Bar-Hillel (1983), Klyve and Lauren (2011), Vivian (2013)). The expected value of a lottery is accepted as the average per game expected monetary value. The run of $N$ games corresponds to a compound lottery $L_{N}$, with the expected monetary value for all $N$ repeated games $N\left(1+\log _{2} N\right)$, as is explained in the paper by Vivian (2013). This gives the average per game expected value

$$
U_{N}=1+\log _{2} N
$$

in some monetary units. This value $U_{N}$ increases with $N$, although much slower than $U_{n}=n$ in the single Bernoulli game (Tversky and Bar-Hillel (1983), Klyve and Lauren (2011), Vivian (2013)). Nevertheless, the expected value $U_{N}$ tends to infinity with $N$.

Similarly to the single game, we have to remember that the expected value $U_{N}$ does not need to equal the willingness to pay $U_{N_{\text {opt }}}$. Then one can ask the question: which of the runs would be preferred by decision makers, that is, what would be the willingness to pay? In other words, how many runs $N$ would be considered by subjects as optimal? 
Following the approach of Sec. 5, the probability of a lottery $L_{N}$ of $N$ repeated games is given by the expression

$$
p\left(L_{N}\right)=\frac{U_{N} e^{\beta U_{N}}}{\sum_{N=0}^{\infty} U_{N} e^{\beta U_{N}}},
$$

with the value $U_{N}$ defined in (53). Here the parameter $\beta<0$ is different from that in probability (24). The stochastically preferred $L_{N}$ is that which is prescribed by the maximum of probability (54), corresponding to the subset consisting of $N_{\text {opt }}$ repeated games. Therefore the willingness to pay is

$$
U_{N_{o p t}}=\frac{1}{|\beta|} .
$$

This defines the optimal number of repeated games by the equation

$$
N_{\text {opt }}=2^{1 /|\beta|-1} .
$$

Overall, the situation is treated similarly to the previous consideration. In the original Bernoulli game, the lotteries $L_{n}$ are characterized by $U_{n}=n$ increasing to infinity with rising $n$. However the stochastically preferred is a lottery $L_{n_{o p t}}$ with a finite $n=n_{\text {opt }}$ given by $1 /|\beta|$. For repeated games, the compound lotteries $L_{N}$ of $N$ repeated games correspond to the values $U_{N}=1+\log _{2} N$ increasing with increasing $N$. However, the optimal lottery, with the optimal number of runs $N_{\text {opt }}$, is described by a finite $N=N_{\text {opt }}$ given by (156).

\section{Inverse St. Petersburg Paradox}

There exists a situation that in some sense is inverse to the St. Petersburg paradox, when one plays a game resulting in losses, whose expected values or expected utilities are negative and tend to $-\infty$. Nevertheless, some people, of course not all but maybe a small fraction, still continue playing. A typical example concerns a roulette. Let us keep in mind the often used roulette whose wheel has 38 numbers, which consist of the numbers one through 36 , the number zero, and the number double zero. The zero and double zero are losing numbers for the players. Thus the odds are in favor of the casino when betting on roulette. Half of the numbers between 1 and 36 are red, and the other half are black. It is possible to place a bet just on a number, on a number being red or black, odd or even, first 18 or last 18, and other combinations.

There is a well-known so-called martingale strategy, used by gamblers which appears to guarantee a win. Every time a player loses, he/she doubles his/her bet on the next spin of the wheel and continuing to do so on each successive loss, when eventually he/she does win. If so, he/she will win back the amount he/she lost in addition to a profit equal to the original stake. However, this strategy leads to consecutive losses rising to infinity. Then the question is: How would it be possible to explain this paradox, when some people keep gambling and losing, instead of stopping?

As an illustration, we can take the example considered by Aloysius (2003), when the player bets on colour or on first/last 18 numbers. The process is assumed to terminate when the gambler wins. The expected value at the $n$-th stage, after the $n$-th spin of the wheel, is

$$
U_{n}=\sum_{k=0}^{n-1} p(1-p)^{k} x_{0}+(1-p)^{n} x_{n}
$$


where $x_{0}$ is the initial bid, $x_{n}$ is the loss accumulated after the $n$-th step, and

$$
x_{n}=-\sum_{k=0}^{n-1} 2^{k} x_{0}, \quad p=\frac{18}{38} .
$$

The values of $U_{n}$ are negative and decreasing, so that

$$
\lim _{n \rightarrow \infty} U_{n}=-\infty
$$

Aloysius (2003) shows that it is possible to find such utility functions $u(x)$ that the expected utility

$$
\widetilde{U}_{n}=\sum_{k=0}^{n-1} p(1-p)^{k} u\left(x_{0}\right)+(1-p)^{n} u\left(x_{n}\right)
$$

enjoys the property

$$
\lim _{n \rightarrow \infty} \widetilde{U}_{n} \geq u(0)
$$

Then for the people characterized by such special utility functions, it looks reasonable to continue playing.

This explanation is similar to that used for making the expected utility finite by introducing concave utility functions in Sec. 3.1 when considering the St. Petersburg paradox. However, as has been explained in that section, it is easy to change the payoffs so that the problem reduces back to the initial formulation with the infinite expected utility. In the same way, here it is possible to change the payoffs $x$ to $y(x)$ such that $u(y(x))=x$. Then expression (60) returns back to (57) and the problem reappears. As before, it is interesting to pose a more general question: Why some people continue gambling despite the increasing losses tending to infinity?

The general answer is as follows. The case of gambling is different from the choice between the lotteries in the St. Petersburg paradox. In the latter, when hesitating what ticket to buy, one considers a set of possible lotteries $L_{n}$, choosing the most preferable among them. In the martingale roulette game, one never considers all possibilities at once, imagining numerous forthcoming losses, but at each step the gambler compares just two alternatives: to stop or keep playing. This choice repeats at each next step. The probability of stopping or playing is given by the probability $p\left(L_{n}\right)$, as discussed in Sec. 4. Suppose one chooses between the alternatives $L_{n}$ and $L_{n+1}$. Despite that the corresponding expected utilities are negative and decreasing, so that $U_{n+1}<U_{n}$, the probabilities $p_{1}\left(L_{n}\right)$ (stop gambling) and $p_{2}\left(L_{n+1}\right)$ (continue gambling) are finite, which means that at each step there are some people deciding to stop, as well as there is a fraction of subjects deciding to keep gambling.

Let us take the expected value (57) that equals the expected utility under a linear utility function. This expected value, by summing the terms, can be rewritten as

$$
U_{n}=\left[1-2^{n}(1-p)^{n}\right] x_{0} .
$$

For $p=18 / 38$, this takes the form

$$
U_{n}=\left[1-\left(\frac{20}{19}\right)^{n}\right] x_{0} .
$$


After the first spin of the roulette wheel, there is the choice either to stop playing, having the expected value $U_{1}=-0.0526 x_{0}$ or to continue gambling getting the value $U_{2}=-0.108 x_{0}$. The probabilities of alternatives with negative expected utilities are given by expression (20). For simplicity, let us accept the case of neutral beliefs, when $\beta=0$. For finite $\beta$, the situation is qualitatively similar. Then we find $p_{1}\left(L_{1}\right)=0.671$ and $p_{2}\left(L_{2}\right)=0.328$. This implies that, despite the loss, about $33 \%$ of gamblers continue gambling.

After the second spin of the wheel, one needs to compare the alternatives $L_{2}$, with $U_{2}=$ $-0.108 x_{0}$, and $L_{3}$, with $U_{3}=-0.166 x_{0}$, which gives $p_{1}\left(L_{2}\right)=0.606$ and $p_{2}\left(L_{3}\right)=0.394$. At the next stage, one compares the alternatives $L_{3}$ and $L_{4}$, with the values $U_{3}=-1.166 x_{0}$ and $U_{4}=-0.228 x_{0}$, which yields $p_{1}\left(L_{3}\right)=0.579$ and $p_{2}\left(L_{4}\right)=0.421$. Then, comparing the alternatives $L_{4}$ and $L_{5}$, with $U_{4}=-0.228 x_{0}$ and $U_{5}=-0.292 x_{0}$, one gets $p_{1}\left(L_{4}\right)=0.562$ and $p_{2}\left(L_{5}\right)=0.438$. For large $n$, such that $n \gg 40$, one has

$$
U_{n} \simeq-\left(\frac{20}{19}\right)^{n} x_{0} .
$$

Then the probabilities of stopping or continuing the roulette gamble tend to

$$
p_{1}\left(L_{n}\right)=0.513, \quad p_{2}\left(L_{n+1}\right)=0.487 \quad(n \gg 40) .
$$

This means that about $49 \%$ of people, who have reached the $n$-th step, decide to keep gambling despite permanent losses.

\section{Conclusion}

In order to resolve the Bernoulli St. Petersburg paradox, there is no need to change the definitions of either utility functions or expected utilities. The fact that the expected utility of the Bernoulli game, in the limit, can be infinite is unavoidable. The Bernoulli game is mathematically correct and defines a bone-fide lottery.

The paradox arises because the lottery with a maximal expected utility corresponds to infinite price, although the majority of real human beings prefer a lottery that does not cost more than one or two tosses. This means that people does not consider as optimal a lottery with a maximal expected utility. That is, the paradox is in the incompatibility of the definition of an optimal lottery as that for which the expected utility is maximal and the behavior of real humans.

However, in the frame of the probabilistic approach, defining a stochastically optimal lottery as that one corresponding to the maximal probability distribution, there is no any paradox, just because a lottery with infinite expected utility is not stochastically optimal. On the contrary, a stochastically optimal is a lottery with a rather low utility corresponding, for real humans, to a couple of tosses.

\section{Acknowledgment}

The author is grateful for discussions to D. Sornette and E.P. Yukalova.

This research did not receive any specific grant from funding agencies in the public, commercial, or not-for-profit sectors. 


\section{References}

Aloysius, J.A. (2003): "Rational escalation of costs by playing a sequence of unfavorable gambles: the martingale", Journal of Economic Behavior and Organization, 51 (2003) 111-129.

Arieli, A., A. Sterkin, A. Grinvald, and A. Aertsen (1986): "Dynamics of ongoing activity: explanation of the large variability in evoked cortical responses", Science, 273, 1868-1871.

Bernoulli, D. (1738): "Exposition of a new theory on the measurement of risk", Proceedings of the Imperial Academy of Sciences of St. Petersburg, 5, 175-192. Reprinted in (1954): Econometrica, 22, 23-36.

Blavatskyy, P. (2005): "Back to the St. Petersburg paradox?" Management Science, 51, 677678.

Blavatskyy, P. (2006): "Violations of betweenness or random errors?", Economics Letters, 91, $34-38$.

Bottom, W.P., R.N. Bontempo, and D.R. Holtgrave (1989): "Experts, novices, and the St. Petersburg Paradox: Is one solution enough?", Journal of Behavioral Decision Making, 2, 113121.

Burnham, K.P., and D.R. Anderson (2002): Model Selection and Multi-Model Inference. Berlin: Springer.

Carbone, E. (1997): "Investigation of stochastic preference theory using experimental data", Economics Letters, 57, 305-311.

Clark, M. (2002): "The St. Petersburg paradox", in Paradoxes from A to Z. London: Routledge, p. 174-177.

Cover, T.M., and J.A. Thomas (2006): Elements of Information Theory. New York: Wiley.

Cox, J.C., V. Sadiraj, and B. Vogt (2009): "On the empirical relevance of St. Petersburg lotteries", Economics Bulletin, 29, 214-220.

Cox, J.C., E.B. Kroll, M. Lichters, V. Sadiraj, and B. Vogt (2018): "The St. Petersburg paradox despite risk-seeking preferences: an experimental study", Business Research, 12, 27-44.

Cramer, G. (1728): "Letter to Nicolas Bernoulli", London, 21 May 1728.

(http://www.cs.xu.edu/math/Sources/Montmort/stpetersburg.pdf\#search=\%22Nicolas\%20Bernoulli

Dmitruk, A.V., and N.V. Kuzkina (2005): "Existence theorem for optimal control problems on an infinite time interval", Mathematical Notes, 78, 466-480.

Fechner, G. (1860): Elements of Psychophysics. New York: Holt, Rinehart and Winston.

French, S., and D.R. Insua (2000): Statistical Decision Theory. London: Arnold.

Gelfand, I.M., and S.V. Fomin (1963): Calculus of Variations. London: Prentice Hall.

Gerken, J. (1991): "Generalized logit model", Transportation Research B, 25, 75-88.

Ghahramani, S. (2000): Fundamentals of Probability. New Jersey: Prentice Hall. 
Glimcher, P.W. (2005): "Indeterminacy in brain and behavior", Annual Review of Psychology, $56,25-56$.

Glimcher, P.W., and A.A. Tymula (2018): "Expected subjective value theory (ESVT): A representation of decision under risk and certainty", University of Sydney Economics Working Paper Series.

Gold, J.I., and M.N. Shadlen (2001): "Neural computations that underlie decisions about sensory stimuli", Trends in Cognitive Science, 5, 10-16.

Gul, F., P. Natenzon, and W. Pesendorfer (2014): "Random choice as behavioral optimization", Econometrica, 82, 1873-1912.

Gustason, W. (1994): Reasoning from Evidence. New York: Macmillan College.

Hardin, R. (1982): Collective Action. Baltimore: John Hopkins University.

Harless, D., and C. Camerer (1994): "The predictive utility of generalized expected utility theories", Econometrica, 62, 1251-1289.

Hayden, B.Y., and M.L. Platt (2009): "The mean, the median, and the St. Petersburg paradox", Judgment and Decision Making, 4, 256-272.

Hey, J.D., and C. Orme (1994): "Investigating generalisations of expected utility theory using experimental data", Econometrica, 62, 1291-1326.

Jeffrey, R.C. (1983): The Logic of Decision. Chicago: University of Chicago.

Kahneman, D., and A. Tversky (1979): "Prospect theory: an analysis of decision under risk", Econometrica, 47, 263-291.

Klyve, D., and A. Lauren (2011): "An empirical approach to the St. Petersburg paradox", College Mathematics Journal, 42, 260-264.

Kullback, S., and R.A. Leibler (1951): "On information and sufficiency", Annals of Mathematical Statistics, 22, 79-86.

Kullback, S. (1959): Information Theory and Statistics. New York: Wiley.

Kurtz-David, V., D. Persitz, R. Webb, and D.J. Levy (2019): "The neural computation of inconsistent choice behavior", Nature Communications, 10, 1583.

Loomes, G., and R. Sugden (1995): "Incorporating a stochastic element into decision theories", European Economic Review, 39, 641-648.

Loomes, G., P. Moffatt, and R. Sugden (2002): "A microeconomic test of alternative stochastic theories of risky choice", Journal of Risk and Uncertainty, 24, 103-130.

Luce, R.D. (1958): "A probabilistic theory of utility", Econometrica, 26, 193-224.

Luce, R.D. (1959): Individual Choice Behavior: A Theoretical Analysis. New York: Wiley.

Luce, R.D. (1977): "The choice axiom after twenty years", Journal of Mathematical Psychology, $15,215-233$.

MacKay, D.J.C. (2003): Information Theory, Inference, and Learning Algorithms. Cambridge: Cambridge University Press. 
Martin, R.M. (2008): "The St. Petersburg paradox", in Stanford Encyclopedia of Philosophy. Stanford University: Stanford.

Menger, K. (1967): "The role of uncertainty in economics", in Essays in Mathematical Economics in Honor of Oscar Morgenstern, edited by M. Shubik. Princeton: Princeton University. Moore, A.W. (1990): The Infinite. London: Routledge.

Nathan, A. (1984): "False expectations", Philosophy of Science, 51, 128-136.

Neugebauer, T. (2010): "Moral impossibility in the St Petersburg paradox: A literature survey and experimental evidence", Luxembourg School of Finance Working Paper, 1-43.

Nielsen, R. (2005): Statistical Methods in Molecular Evolution. Berlin: Springer.

Nobandegani, A.S., and T.R. Shultz (2020): "The St. Petersburg paradox: A fresh algorithmic perspective", Association for the Advancement of Artificial Intelligence Working Paper, 1-4.

Pfiffermann, M. (2011): "Solving the St. Petersburg paradox in cumulative prospect theory: the right amount of probability weighting", Theory and Decision, 71, 325-341.

Pinski, F.J., G. Simpson, A.M. Stuart, and H. Weber (2015): "Kullback-Leibler approximation for probability measures on infinite dimensional spaces", SIAM Journal of Mathematical Analysis, 47, 4091-4122.

Poincaré, H. (1902): Science and Hypothesis. London: Walter Scott.

Rayo, L., and G.S. Becker (2007): "Evolutionary efficiency and happiness", Journal of Political Economy, 2007, 115, 302-337.

Rieger, M.O., and M. Wang (2006): "Cumulative prospect theory and the St. Petersburg paradox", Economic Theory, 28, 665-679.

Rivero, J.C., D.R. Holtgrave, R.N. Bontempo, and W.P. Bottom (1990): "The St. Petersburg Paradox: Data, at last". Commentary, 8, 46-51.

Resnik, M.D. (1987): Choices: An Introduction to Decision Theory. Minneapolis: University of Minnesota.

Rubinstein, R.Y., and D.P. Kroese (2004): The Cross-Entropy Method. Berlin: Springer.

Rucker, R. (1995): Infinity and the Mind: The Science and Philosophy of the Infinite. Princeton: Princeton University.

Samuelson, P.A. (1960): "The St. Petersburg paradox as a divergent double limit", International Economic Review, 1, 31-37.

Samuelson, P.A. (1977): "St. Petersburg paradoxes: defanged, dissected, and historically described", Journal of Economic Literature, 15, 24-55.

Savage, L. (1954): The Foundations of Statistics. New York: Wiley.

Schumacher, J.F., S.K. Thompson, and C.A. Olman (2011): "Contrast response functions for single Gabor patches: ROI-based analysis over-represents low-contrast patches for GE BOLD", Frontiers in System Neuroscience 5, 1-10. 
Seidl, C. (2013): "The St. Petersburg Paradox at 300", Journal of Risk and Uncertainty, 46, $247-264$.

Shadlen, M.N. and D. Shohamy (2016): "Perspective decision making and sequential sampling from memory", Neuron, 90, 927-939.

Shore, J., and R. Johnson (1980): "Axiomatic derivation of the principle of maximum entropy and the principle of minimum cross-entropy", IEEE Transactions on Information Theory, 26, 26-37.

Slovic, P., C.K. Mertz, D.M. Markowitz, A. Quist, and D. Västfjäll (2020): "Virtuous violence from the war room to death row", Proceedings of the National Academy of Sciences of USA 117, 20474-20482.

Small, K. (1987): "A discrete choice model for ordered alternatives", Econometrica, 55, 409424.

Tversky, A., and M. Bar-Hillel (1983): "Risk: The long and the short", Journal of Experimental Psychology: Learning, Memory, and Cognition, 9, 713-717

Vivian, R.W. (2004): "Simulating Daniel Bernoulli's St. Petersburg game: Theoretical and empirical consistency". Simulation and Gaming, 35, 499-504.

Vivian, R.W. (2013): "Ending the myth of the St. Petersburg paradox", South African Journal of Economic and Management Sciences, 16, 347-364.

von Neuman, J., and O. Morgenstern (1944): Theory of Games and Economic Behavior. Princeton: Princeton University.

Webb, R. (2019): "The neural dynamics of stochastic choice", Management Science, 65, 230255.

Weirich, P. (1984): "The St. Petersburg gamble and risk", Theory and Decision, 17, 193-202.

Wen, C.H., and F.S. Koppelman (2001): "The generalized nested logit model", Transportation Research B, 35, 627-641.

Werner, G., and V.B. Mountcastle (1963): "The variability of central neural activity in a sensory system and its implications for the central reflection of sensory events", Journal of Neurophysiology, 26, 958-977.

Williams, R. (2016): "Understanding and interpreting generalized ordered logit models", Journal of Mathematical Sociology, 40, 7-20.

Woodford, M. (2020): "Modeling imprecision in perception, valuation, and choice", Annual Review of Economics, 12, 579-601.

Yukalov, V.I., and D. Sornette (2014): "Role of information in decision making of social agents", International Journal of Information Technology and Decision Making, 14, 1129-1166.

Yukalov, V.I., and D. Sornette (2018): "Quantitative predictions in quantum decision theory", IEEE Transactions on Systems, Man and Cybernetics Systems, 48, 2168-2216. 